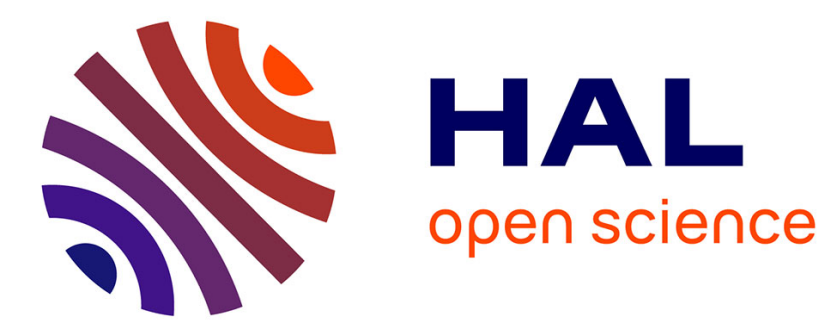

\title{
Couplage entre alignement et orientation par collisions en champ magnétique intense
}

\author{
J.-C. Gay
}

\section{To cite this version:}

J.-C. Gay. Couplage entre alignement et orientation par collisions en champ magnétique intense. Journal de Physique Lettres, 1975, 36 (10), pp.239-242. 10.1051/jphyslet:019750036010023900 jpa00231197

\section{HAL Id: jpa-00231197 https://hal.science/jpa-00231197}

Submitted on 1 Jan 1975

HAL is a multi-disciplinary open access archive for the deposit and dissemination of scientific research documents, whether they are published or not. The documents may come from teaching and research institutions in France or abroad, or from public or private research centers.
L'archive ouverte pluridisciplinaire HAL, est destinée au dépôt et à la diffusion de documents scientifiques de niveau recherche, publiés ou non, émanant des établissements d'enseignement et de recherche français ou étrangers, des laboratoires publics ou privés. 


\title{
COUPLAGE ENTRE ALIGNEMENT ET ORIENTATION PAR COLLISIONS EN CHAMP MAGNÉTIQUE INTENSE
}

\author{
J.-C. GAY \\ Laboratoire de Spectroscopie Hertzienne de l'ENS (*) et Université Paris VII \\ 4, place Jussieu, Tour 12, 1er étage, 75230 Paris Cedex 05, France
}

(Reçu le 16 juin 1975, accepté le 8 juillet 1975)

\begin{abstract}
Résumé. - Un modèle théorique simple nous permet de montrer qu'il peut se créer de l'orientation dans une vapeur alignée, lors de collisions en champ magnétique intense. Il suffit pour cela que la précession de Larmor de l'état excité des atomes ne soit pas négligeable pendant la durée de la collision. L'ordre de grandeur de l'effet est donné pour un potentiel de Van der Waals en $R^{-6}$ et une possibilité de mise en évidence expérimentale est décrite.
\end{abstract}

\begin{abstract}
Using a simple theoretical model we predict the occurrence of orientation in an aligned vapour when the atoms undergo collisions in a high magnetic field. The main condition for this to occur is that the Larmor precession of the excited states of the atoms is not negligible during the collision. We give the order of magnitude of the effect for a $R^{-6}$ Van der Waals interaction and we describe possible conditions for observing it experimentally.
\end{abstract}

1. Introduction. - L'étude théorique de l'action d'un champ magnétique intense sur les processus de collision en phase vapeur fait apparaitre deux catégories d'effets nouveaux par rapport à la situation où le champ est nul :

1.1 Les taux de relaxation ou de transfert par collision dépendent de l'intensité du champ.

1.2 Les symétries de la matrice de relaxation sont modifiées. Sous certaines hypothèses, la matrice de relaxation présente la symétrie sphérique en champ nul. Il n'en est plus de même en présence d'un champ magnétique qui privilégie une direction de l'espace.

Ces effets ne sont évidemment notables que pour des champs magnétiques tels que l'évolution atomique qu'ils produisent pendant la durée de la collision soit appréciable [1].

Nous présentons ci-dessous l'illustration de l'un des aspects du point 2 dans le cas de collisions entre un atome excité et un perturbateur sans structure avec la possibilité d'orienter par collisions en champ intense, une vapeur alignée.

Nous montrons pour un niveau excité $J=1$ et une interaction en $R^{-6}$ du type Van der Waals que les probabilités de transferts entre les sous-niveaux Zeeman $m=0$ et $m= \pm 1$ sont différentes, à l'aide d'un calcul de perturbation au $3^{\mathrm{e}}$ ordre de l'interaction.

Nous donnons ensuite, afin d'évaluer l'ordre de

(*) Associé au C.N.R.S. No 18. grandeur de l'effet, les taux de transfert par collision entre les sous-niveaux Zeeman. Ces résultats sont obtenus par intégration numérique de l'équation de Schrödinger et seront décrits plus en détail dans une prochaine publication. Ceci nous permet de discuter brièvement les possibilités de mise en évidence expérimentale de l'effet.

2. Hypothèses du modèle. - On se limite à des situations où la différence d'énergie entre les sousniveaux Zeeman est petite devant $k T$. On considère des atomes dans l'état excité $J=1$. Les perturbateurs sont dans l'état fondamental que l'on suppose à symétrie sphérique et sans structure. La collision est traitée dans l'approximation d'impact à trajectoire classique rectiligne. Nous supposons l'isotropie dans l'espace de la distribution des paramètres d'impact et des vitesses relatives. La partie isotrope du potentiel d'interaction qui ne présente d'intérêt que pour le calcul de l'élargissement et du déplacement des raies optiques est ignorée. La partie anisotrope du potentiel a la symétrie d'un alignement dans la direction interatomique.

L'effet des collisions est décrit dans une base standard liée au champ magnétique. Le trièdre de collision lié à une collision particulière est tel que $O x$ soit parallèle au paramètre d'impact $\mathbf{b}, O y$ à la vitesse relative $\mathbf{v}$ et $O z$ perpendiculaire au plan de la collision.

Il se déduit d'un repère fixe de l'espace où $O z$ est parallèle au champ magnétique par la rotation $\mathcal{R}^{-1}(\varphi, \theta, \gamma)$ où $(\varphi, \theta, \gamma)$ sont les angles d'Euler [2]. 
Le principe du calcul est d'évaluer l'effet d'un collision définie par $(\varphi, \theta, \gamma)$ sur l'état excité de l'atome, compte tenu de son évolution sous l'influence du champ magnétique, et d'effectuer la moyenne angulaire sur toutes les orientations possibles dans l'espace du paramètre d'impact et de la vitesse relative.

3. Calcul asymptotique des probabilités de transfert. - Nous emploierons les notations suivantes :

$$
\begin{aligned}
q & =\frac{p}{b^{5} v} \quad \eta=\frac{\omega b}{v}=\frac{\tau}{q^{1 / 5}} \\
\tau & =\frac{\omega \cdot p^{1 / 5}}{v^{6 / 5}}
\end{aligned}
$$

où $\omega, p, v$ sont respectivement la fréquence de Larmor, la force du potentiel et la vitesse relative; le sens de ces notations a déjà été explicité dans une précédente publication, dans le cas d'un potentiel en $R^{-3}$ [3].

La partie anisotrope du potentiel s'écrit sous lá forme :

$$
V(x)=q \sum_{q_{1}}(-)^{q_{1}} U_{-q_{1}}^{2}(\varphi, \theta, \gamma, x) \mathbf{T}_{q_{1}}^{2}
$$

où $\mathbf{T}_{q_{1}}^{2}$ est un tenseur irréductible de rang 2 construit sur la base standard liée à $\mathbf{H}$ et

$$
U_{q_{2}}^{2}(\varphi, \theta, \gamma, x)=\sum_{q_{1}} u_{q_{1}}^{2}(x) R_{q_{1} q_{2}}^{(2)}(\varphi, \theta, \gamma)
$$

dans le cas d'un potentiel en $R^{-6}$ :

$$
\begin{aligned}
& u_{ \pm 2}^{2}=(1 \pm i x)^{2} / 2 r^{8} \\
& u_{ \pm 1}^{2}=0 \\
& u_{0}^{2}=-1 / \sqrt{6} r^{6}
\end{aligned}
$$

avec

$$
r^{2}=1+x^{2} \quad \text { et } \quad x=v t / b
$$

$(\varphi, \theta, \gamma)$ sont les angles d'Euler caractérisant une collision particulière.

En renrésentation d'interaction par rapport au hamiltonien Zeeman, l'équation de Schrödinger s'écrit $(\hbar=1)$ :

$$
i \frac{\mathrm{d}}{\mathrm{d} x}|\psi(x)\rangle=H(x)|\psi(x)\rangle
$$

avec

$$
H(x)=\mathrm{e}^{i \eta J_{H} x} V(x) \mathrm{e}^{-i \eta J_{H} x} .
$$

Notant $S(\varphi, \theta, \gamma)$ la matrice de collision associée à la collision définie par les angles d'Euler $(\varphi, \theta, \gamma)$, on définit les probabilités de transfert entre les sous- niveaux Zeeman pour un paramètre d'impact, une vitesse relative et une valeur du champ magnétique de la manière usuelle :

$$
\Pi^{(m m)(p p)}=-\left\{|\langle m|S| p\rangle|^{2}\right\}
$$

où les crochets désignent la moyenne angulaire à effectuer sur l'ensemble des orientations de $\mathbf{b}$ et $\mathbf{v}$.

On développe la matrice $S(\varphi, \theta, \gamma)$ au $3^{\mathrm{e}}$ ordre de l'interaction. Le développement comporte donc 4 termes

$$
S^{\mathrm{AS}}=I+A(q)+B\left(q^{2}\right)+C\left(q^{3}\right) .
$$

Un tel développement permet d'obtenir les coefficients $\Pi^{(m m)(p p)}$ au $3^{\mathrm{e}}$ ordre en $q$ inclus.

Le premier terme non nul est du second ordre en $q$. Il correspond formellement aux termes $A A^{\dagger}, B, B^{\dagger}$ de l'expression (5). Des considérations de symétrie permettent de prévoir a priori qu'il est le même pour $p=0, m= \pm 1$ et $p= \pm 1, m=0$.

On obtient

$$
\begin{array}{r}
\Pi^{(00)( \pm 1 \pm 1)}\left(q^{2}\right)=\Pi^{( \pm 1 \pm 1)(00)}\left(q^{2}\right)=-\frac{S(\eta)}{10} \\
S(\eta)=\frac{\pi^{2} \cdot \mathrm{e}^{-2 \eta}}{(48)^{2}} q^{2}\left(4 \eta^{6}+24 \eta^{5}+78 \eta^{4}+168 \eta^{3}+\right. \\
\left.+252 \eta^{2}+252 \eta+126\right)
\end{array}
$$

Cette expression a déjà été trouvée par Tsao et Curnutte [4] lors de l'analyse d'un problème analogue.

Nous allons montrer que le terme d'ordre 3 en $q$ qui est nul lorsque $\eta=0$ (cas du champ nul), ne l'est plus dès que le champ $H$ diffère de la valeur 0 , et qu'il contribue avec des signes opposés aux probabilités de transfert $\Pi^{(00)(11)}$ et $\Pi^{(00)(-1-1)}$.

Les termes contribuant à l'ordre 3 en $q$ sont $B A^{\dagger}$, $A B^{\dagger}, C, C^{\dagger}$. Lorsque $m$ diffère de $p$, les termes en $C$ et $C^{\dagger}$ donnent une contribution nulle.

Il reste donc :

$$
\Pi^{(m m)(p p)}\left(q^{3}\right)=2 \operatorname{Re}\{\langle m|B| p\rangle\langle p|A| m\rangle\}
$$

avec

$$
\begin{aligned}
& A=-i \int_{-\infty}^{+\infty} H(x) \mathrm{d} x \\
& B=-\int_{-\infty}^{+\infty} \int_{-\infty}^{x^{\prime}} H\left(x^{\prime}\right) H\left(x^{\prime \prime}\right) \mathrm{d} x^{\prime} \cdot \mathrm{d} x^{\prime \prime} .
\end{aligned}
$$

Les moyennes angulaires dans (6) sont effectuées en utilisant la formule (2) et la relation

$$
\left\{R_{q_{0}^{\prime}-q_{0}}^{(2)} R_{q_{1}^{\prime}-q_{1}}^{(2)} R_{q_{2}^{\prime}-q_{2}}^{(2)}\right\}=\frac{(-)^{q_{0}+q_{0}^{\prime}}}{5}\left\langle 22 q_{1}^{\prime} q_{2}^{\prime} \mid 2-q_{0}^{\prime}\right\rangle\left\langle 22-q_{1}-q_{2} \mid 2 q_{0}\right\rangle .
$$


On obtient alors :

$$
\begin{aligned}
\Pi^{(m m)(p p)}\left(q^{3}\right)=i \sum_{\substack{q_{0} q_{1} q_{2} \\
q_{0} q_{1}^{\prime} q_{2}^{\prime}}} \frac{(-)^{q_{1}+q_{2}-q_{0}^{\prime}}}{5}\left\langle p\left|\mathbf{T}_{q_{0}}^{2}\right| m\right\rangle\left\langle m\left|\mathbf{T}_{q_{1}}^{2} \mathbf{T}_{q_{2}}^{2}\right| p\right\rangle\left\langle 22 q_{1}^{\prime} q_{2}^{\prime} \mid 2-q_{0}^{\prime}\right\rangle \times \\
\times\left\langle 22-q_{1}-q_{2} \mid 2 q_{0}\right\rangle J_{q_{0}^{\prime}}\left(\eta q_{0}\right)\left\{\Phi_{q_{1}^{\prime} q_{2}}\left(\eta q_{1}, \eta q_{2}\right)-\Phi_{q_{1}^{\prime} q_{2}^{\prime}}^{*}\left(\eta q_{1}, \eta q_{2}\right)\right\}
\end{aligned}
$$

avec

$$
\begin{gathered}
\Phi_{q_{1}^{\prime} q_{2}^{\prime}\left(\eta_{1} \eta_{2}\right)}=\int_{-\infty}^{+\infty} \int_{-\infty}^{x^{\prime}} \mathrm{d} x^{\prime} \cdot \mathrm{d} x^{\prime \prime} \mathrm{e}^{i \eta_{1} x^{\prime}+i \eta_{2} x^{\prime \prime}} u_{q_{1}^{\prime}}^{2}\left(x^{\prime}\right) u_{q_{2}}^{2}\left(x^{\prime \prime}\right) \\
J_{q_{0}}(\eta)=\int_{-\infty}^{+\infty} \mathrm{e}^{i \eta x} u_{q_{0}}^{2}(x) \cdot \mathrm{d} x .
\end{gathered}
$$

Il reste à prouver que ce terme est non nul. Son évaluation analytique complète dans le cas d'un potentiel en $R^{-6}$ parait difficile à mener à bien. Nous nous bornerons donc à effectuer un développement limité au $1^{\text {er }}$ ordre en $\eta$. Le terme d'ordre 0 en $\eta$ est nul. L'évaluation des intégrales doubles dans le terme du $1^{\mathrm{er}}$ ordre en $\eta$ ne présente pas de difficultés. On obtient :

$$
\begin{aligned}
& \Pi^{(m m)(p p)}\left(q^{3}\right)=-\sqrt{\frac{7}{3}} \frac{\pi^{2}}{(16)^{3}} \eta q^{3} \sum_{q_{0} q_{1} q_{2}}(-)^{q_{0}} \times \\
& \quad \times\left\langle 22-q_{1}-q_{2} \mid 2 q_{0}\right\rangle\left(q_{2}-q_{1}\right)\left\langle p\left|\mathbf{T}_{q_{0}}^{2}\right| m\right\rangle \\
& \quad \times\left\langle m\left|\mathbf{T}_{q_{1}}^{2} \mathbf{T}_{q_{2}}^{2}\right| p\right\rangle .
\end{aligned}
$$

En prenant le complexe conjugué de cette expression réelle et en changeant les indices $q_{i}$ en leurs opposés, on démontre la relation $\Pi^{(m)(p p)}=\Pi^{(p p)(m m)}$ que l'on peut obtenir par des considérations plus générales de symétrie sur la matrice de relaxation [1]. Le changement global des signes de $m$ et de $p$ et des indices $q_{i}$ joint à quelques transformations simples des coefficients de Clebsch-Gordan conduit à la relation :

$$
\Pi^{(m m)(p p)}\left(q^{3}\right)=-\Pi^{(-m-m)(-p-p)}\left(q^{3}\right) .
$$

L'expression de la correction du $3^{\mathrm{e}}$ ordre en $q$ aux probabilités de transfert s'écrit finalement :

$$
\begin{aligned}
\Pi^{(11)(00)} & \left(q^{3}\right)=\Pi^{(00)(11)}\left(q^{3}\right)= \\
& =-\Pi^{(-1-1)(00)}\left(q^{3}\right)=-\Pi^{(00)(-1-1)}\left(q^{3}\right) \\
& =\frac{7}{4(16)^{3}} \pi^{2} \eta q^{3}
\end{aligned}
$$

La relation (7) qui révèle que les taux de transfert du niveau $m=0$ vers les niveaux $m= \pm 1$ sont différents prouve qu'il existe un terme de couplage non nul entre orientation et alignement longitudinaux défini par $\Pi_{0}^{12}=\Pi_{0}^{21}=-\operatorname{Tr}\left\{T_{0}^{1 \dagger} S T_{0}^{2} S^{\dagger}\right\}$ et dont le développement asymptotique est

$$
\Pi_{0}^{12} \simeq-\frac{7 \sqrt{3}}{4(16)^{3}} \pi^{2} \eta q^{3} .
$$

La création par excitation optique par exemple d'un alignement dans l'état excité de l'atome entraî- nera donc la création d'une orientation sous l'effet combiné des collisions et du champ magnétique. De plus, le sens de l'orientation créée, c'est-à-dire le signe de la différence de population en régime stationnaire entre les niveaux $m= \pm 1$ dépend du signe de la partie anisotrope du potentiel. Si cette partie du potentiel est attractive pour les niveaux $\Sigma$ de la pseudo-molécule, le niveau $m=+1$ sera moins peuplé que le niveau $m=-1$.

Remarque. Les résultats qui précèdent ne dépendent pas en définitive de la forme explicite adoptée pour les variations du potentiel avec la distance interatomique, mais seulement des propriétés de symétrie de celui-ci. Il suffit en effet que la partie anisotrope du potentiel ait le caractère d'un alignement le long de l'axe internucléaire (les éléments de symétrie d'un champ électrique) pour que l'on puisse prévoir le résultat par des considérations de symétrie sur la matrice de collision et sur la matrice de relaxation [1].

4. Ordre de grandeur de l'effet. - Par intégration numérique de l'équation de Schrodinger nous avons obtenu des résultats qui seront publiés prochainement [1] et que nous résumons ci-dessous. Nous donnons sur la figure 1 le comportement des coefficients $\Pi^{(11)(00)}$ et $\Pi^{(-1-1)(00)}$ en fonction de $q^{-2 / 5}\left(q^{-2 / 5}\right.$ est proportionnel au carré du paramètre d'impact). Pour $q<2$, les résultats sont en bon accord avec ceux de la formule approchée (7). On peut noter que la différence entre $\Pi^{(11)(00)}$ et $\Pi^{(-1-1)(00)}$ se manifeste à longue distance pour $q \simeq 1$ lorsque le paramètre d'impact est de l'ordre du rayon de Weisskopf, et que l'hypothèse d'un potentiel en $R^{-6}$ dans cette région n'est pas a priori dénuée de sens.

Le calcul de la matrice de relaxation après intégration numérique du système de collision et sommation sur le paramètre d'impact permet d'obtenir une évaluation des effets précédents : les variations avec le champ des taux de transfert $g^{(m m)(p p)}$ entre les sousniveaux Zeeman sont données sur la figure 2 . Il y apparaît que l'écart entre les taux de transfert de $m=0$ vers $m= \pm 1$ peut atteindre $30 \%$ dans ce modèle et par suite qu'une mise en évidence expérimentale n'est pas exclue. De plus, la relative impor- 


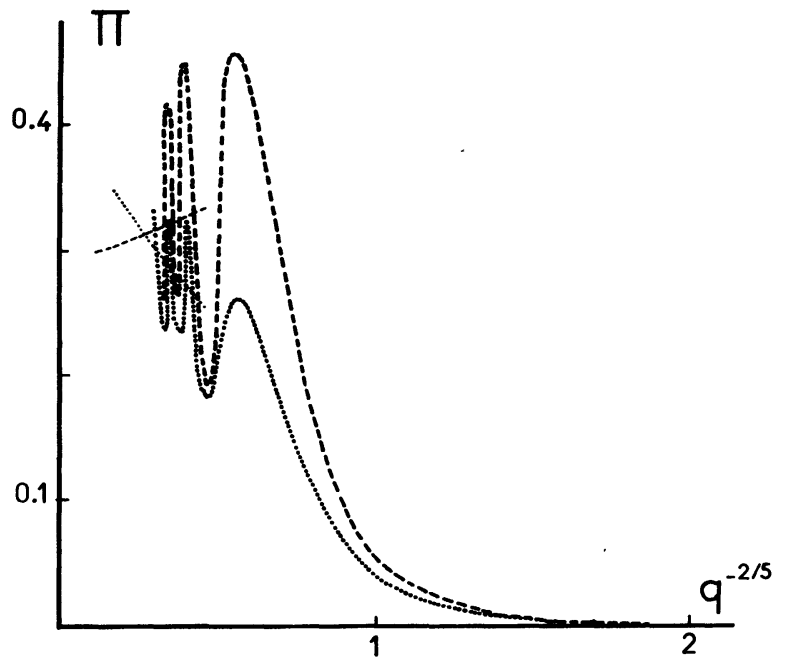

Fig. 1. - Variations en fonction de $q^{-2 / 5}$ (proportionnel au carré du paramètre d'impact) des probabilités de transfert par collision entre les sous-niveaux Zeeman $m=0$ et $m= \pm 1$, pour $\tau$ égal à 1 :

$$
--\left|\Pi^{(-1-1)(00)}\right| ; \ldots\left|\Pi^{(11)(00)}\right| \text {. }
$$

tance de l'effet prévu permet de supposer que certaines approximations telles celles de trajectoires rectilignes ne sont pas cruciales et que l'effet se produisant à longue distance, un traitement quantique de la collision n'est pas nécessaire.

L'allure des courbes données sur la figure 2 appelle pour $\tau>3$ quelques commentaires. L'effet semble en effet s'inverser en champ plus élevé. Ceci peut être compris de la façon suivante : lorsque l'on sépare l'évaluation de la section efficace en deux régions $q$ supérieur ou inférieur à 20 , on constate que ces deux régions contribuent au terme de couplage orientationalignement avec le signe contraire. Lorsque $\tau$ est inférieur à 3 la contribution $q<20$ est largement prédominante mais la situation s'inverse pour des

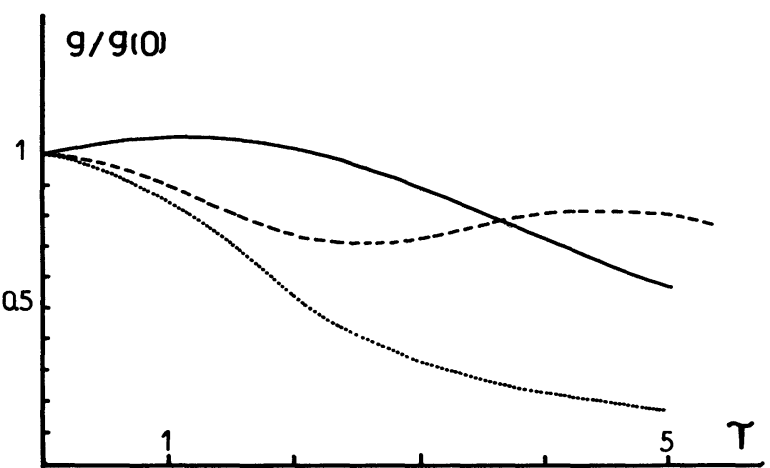

Fig. 2. - Variation des taux de transfert en fonction de $\tau$ (proportionnel au champ magnétique) pour une interaction en $R^{-6}$. Les taux de transfert sont normalisés à leurs valeurs en champ nul. $-g^{(-1-1)(00)} ; \ldots g^{(11)(00)} ; \ldots g^{(11)(-1-1)}$

valeurs de $\tau$ plus grandes. Dans ce cas, la contribution des collisions à courte distance est prépondérante et le modèle en $R^{-6}$ adopté est certainement irréaliste [1].

Le cas des collisions $\mathrm{Hg}^{*}\left(6{ }^{3} \mathrm{P}_{1}\right)$-Xénon semble favorable à une mise en évidence expérimentale de l'effet que nous venons de décrire. L'ordre de grandeur des champs nécessaires peut être évalué à partir des sections de dépolarisation en champ nul [5] et des paramètres atomiques. Le paramètre $\tau$ est de l'ordre de 1 pour un champ de $25 \mathrm{kG}$. Les conditions expérimentales les plus favorables semblent donc se situer entre cette valeur et $100 \mathrm{kG}$.

Remarquons que dans les conditions de la référence [6], la section efficace mesurée correspond à $\frac{1}{2}\left(\sigma^{(11)(00)}+\sigma^{(-1-1)(00)}\right)$. Pour un champ de $60 \mathrm{kG}$, $\tau$ vaut environ 2,4 , et les variations relatives théoriques de $15 \%$ sont en bon accord avec celles obtenues expérimentalement. Néanmoins, cela n'autorise évidemment pas à conclure quant à la validité d'un potentiel en $R^{-6}$ pour décrire de telles collisions.

\section{Bibliographie}

[1] GAY, J.-C., soumis à J. Physique.

[2] Nous adoptons pour les rotations les notations de A. MessiaH, Mécanique Quantique (Dunod) Paris.

[3] Gay, J.-C., J. Physique 35 (1974) 813.

[4] Tsao, C. J. et Curnutte, B., J. Quant. Spectrosc. Radiat. Transfer 2 (1962) 41.
[5] Barrat, J. P., Casalta, D., Cojan, J. L. et Hamel, J., J. Physique 27 (1966) 608.

Faroux, 'J. P., Thèse (Paris) 1969.

[6] Gay, J. C. et SchneIder, W. B., J. Physique Lett. 36 (1975) L-185. 